\title{
Une nouvelle espèce menacée de Euphorbia sect. Goniostema (Euphorbiaceae) du nord de Madagascar
}

\author{
Jean-Philippe Castillon \& Jean-Bernard Castillon
}

\begin{abstract}
CASTILLON, J.-P. \& J.-B. CASTILLON (2018). A new threatened species of Euphorbia sect. Goniostema (Euphorbiaceae) from northern Madagascar. Candollea 73: 187-191. In French, English and French abstracts. DOI: http://dx.doi.org/10.15553/c2018v732a2

Euphorbia longitubicinicyathium J.-P. Castillon \& J.-B. Castillon (Euphorbiaceae), a new species from northeastern Madagascar is described and illustrated with field photographs. The new species belongs to Euphorbia sect. Goniostema Baill. ex Boiss. and is morphologically related to non thorny species of the east coast of Madagascar (e.g. Euphorbia geroldii Rauh, Euphorbia robivelonae Rauh or Euphorbia thuarsiana Baill.) but differs by its larger dimensions, its habit and its long trumpet shaped cyathia.

\section{Résumé}

CASTILLON,J.-P. \& J.-B. CASTILLON (2018). Une nouvelle espèce menacée de Euphorbia sect. Goniostema (Euphorbiaceae) du nord de Madagascar. Candollea 73: 187-191. En français, résumés anglais et français. DOI: http://dx.doi.org/10.15553/c2018v732a2

Euphorbia longitubicinicyatbium J.-P. Castillon \& J.-B. Castillon (Euphorbiaceae), une nouvelle espèce du nord-est de Madagascar est décrite et illustrée par des photos de terrain. Cette nouvelle espèce, appartenant à Euphorbia sect. Goniostema Baill. ex Boiss., est proche morphologiquement des autres espèces dépourvues d'épines de la côte est de Madagascar (par exemple Euphorbia geroldii Rauh, Euphorbia robivelonae Rauh ou Euphorbia thuarsiana Baill.), mais s'en distingue aisément par sa plus grande taille, son port général et ses longues cyathes en forme de trompette.
\end{abstract}

\section{Keywords}

EUPHORBLACEAE - Euphorbia - Euphorbia sect. Goniostema - Madagascar - Taxonomy - New species

\footnotetext{
Adresses of the authors:

JPC: IUT de Saint-Pierre, Université de la Réunion, rue des Capucins 1, 97427 L'Etang-Salé-les-Bains, La Réunion, France.

E-mail: jean-philippe.castillon@univ-reunion.fr

JBC: Professeur retraité de l'Université de La Réunion, rue Jean Albany 41, 97430 Le Tampon, La Réunion, France.
}

Submitted on March 5, 2018. Accepted on August 14, 2018.

First published online on November 5, 2018 


\section{Introduction}

Le genre Euphorbia L. est largement représenté à Madagascar avec environ 170 espèces (AuBRiot et al., 2014) pour la plupart endémiques. Les quatre sous-genres y sont représentés: i.e. Euphorbia subg. Athymalus Neck. ex Rchb., Euphorbia subg. Esula Pers., Euphorbia subg. Chamaesyce Raf. et Euphorbia subg. Euphorbia. Parmi ceux-ci, le sous-genre Euphorbia est de loin le plus diversifié, avec plusieurs sections endémiques (ou quasiendémiques) de Madagascar comme par exemple: Euphorbia sect. Tirucalli Boiss. (Madagascar, Afrique, îles de l'Océan Indien occidental, Yémen, Inde) et Euphorbia sect. Deuterocalli Croizat pour les euphorbes coralliformes; Euphorbia sect. Denisophorbia (Léandri) Croizat (Madagascar, Comores, Île Maurice et Seychelles), Euphorbia sect. Pervilleanae Haev. \& X. Aubriot et Euphorbia sect. Pachysanthae X. Aubriot \& Haev. pour des euphorbes non épineuses arbustives ou arborescentes; Euphorbia sect. Goniostema Baill. ex Boiss. pour les euphorbes à larges cyatophylles colorés, section la plus riche avec environ 77 espèces (Dorsey et al., 2013).

Cette dernière section englobe la totalité des espèces épineuses et/ou géophytes de Madagascar, qui sont aussi les espèces les plus prisées des collectionneurs comme Euphorbia milii Des Moul. ou E. primulifolia Baker. Elle contient aussi certaines espèces arbustives non épineuses, originaires principalement des forêts de la côte est malgache comme $E$. geroldii Rauh, E. robivelonae Rauh, E. thuarsiana Baillon, E. tardieuana Leandri, E. mangelsdorffi Rauh ou E. hexadenia Denis, et l'espèce décrite ci-dessous, E. longitubicinicyathium J.-P. Castillon \& J.-B. Castillon, appartient à ce dernier groupe.

Lors d'une visite au Tsingy Rouge près d'Antsiranana, nous avons trouvé une euphorbe immédiatement identifiée comme nouvelle du fait de sa grande taille, de son port lianescent, et de ses longues cyathes rouges en trompette. Une recherche à TAN nous a permis d'identifier une première récolte de cette nouvelle espèce. De plus, grâce aux localités et aux descriptions des étiquettes de récoltes réalisées par les botanistes du programme de recherche et conservation du Missouri Botanical Garden à Madagascar et aux scans fournis par MO, nous avons pu identifier trois récoltes qui représentent notre nouvelle espèce. Nous fournissons ci-dessous une description de cette nouvelle euphorbe, une discussion sur ses affinités morphologiques, des photos de terrains ainsi que son statut provisoire de conservation suivant les Critères et les Catégories de la Liste Rouge de l'UICN (2012).

\section{Traitement taxonomique}

Euphorbia longitubicinicyathium J.-P. Castillon \& J.-B. Castillon, spec. nova (fig. 1).

Holotypus: Madagascar. Prov. Antsiranana: Cne rurale de Sadjoavato, Andrafiabe, forêt de Sahafary, $12^{\circ} 34^{\prime} \mathrm{S}$ $49^{\circ} 27^{\prime} \mathrm{E}, 180 \mathrm{~m}, 24 . V I I .2004$, Riri Guittou 26 (TAN!; iso-: CNARP, MO-6120385, P).

Haec species generis Euphorbiae sect. Goniostema Baill. ex Boiss. ceteris speciebus affinis est sed, caule usque ad $5 \mathrm{~m}$ alto basique solum 2-5 cm in diametro, foliorum forma coloreque et rubris longis tubicinibusque cyathiis, praecipue differt.

Arbuste pouvant atteindre $5 \mathrm{~m}$ de hauteur, non succulent, non épineux. Tige principale de $2-5 \mathrm{~cm}$ de diam. à la base, plutôt lisse, gris-marron avec des bandes blanches d'environ 8-10 cm de hauteur; ramifications primaires de $1 \mathrm{~cm}$ de diam., irrégulièrement espacées tous les $20-50 \mathrm{~cm}$, dépassant $1 \mathrm{~m}$ de longueur; tiges terminales de 10-20 cm de long $\times 2-3 \mathrm{~mm}$ de diamètre. Feuilles persistantes, allongées, de forme lancéolée, rhomboïdale ou droite, $60 \times 8 \mathrm{~mm}$, disposées en rosettes en forme d'étoile de $6-10$ feuilles de tailles inégales (les premières $10 \times 3 \mathrm{~mm}$ ) à l'extrémité des tiges, lisses, glabres, de couleur unie, vert vif sur le dessus, bien plus pâle, presque blanchâtre, en dessous; apex pointu ou arrondi, prolongé par un mucron blanc récurvé de 1-1,5 mm; marge continue, blanche; nervation pennée, nervure principale claire et légèrement en relief, environ 15-20 nervures secondaires à peine visibles; pétiole court $4 \times 1 \mathrm{~mm}$ de couleur rouge bordeaux. Stipules $2 \times 0,5 \mathrm{~mm}$, fimbriées, de couleur jaune marron, une de chaque côté du pétiole de la feuille, caduques. Incyathescences généralement solitaires, en forme de cyme, en position sub-terminale ou à l'aisselle des nouvelles tiges, formées généralement de deux dichasia, parfois d'un seul; pédoncule commun $14 \times 1 \mathrm{~mm}$, rouge avec à sa base deux bractées rouge foncé, triangulaires, charnues, $2 \times 2 \mathrm{~mm}$; pédoncules secondaires $12 \times 1 \mathrm{~mm}$, rouges, avec deux bractées de $1 \mathrm{~mm}$ à leur base; bractées des cyathes de couleur marron-vert à marge rouge, $3 \times 2 \mathrm{~mm}$, engainantes, à extrémité mucronée. Cyathium hermaphrodite, pendant, en forme de cor, $20 \times 10 \mathrm{~mm}$; pédoncule rouge parfois tacheté de blanc, $10 \mathrm{~mm}$, se prolongeant en deux cyathophylles. Cyathophylles, 2, d'abord plaqués sur l'involucre, puis le dépassant largement (3-5 mm), finalement ouverts et arrondis (10 $\mathrm{mm}$ de diam.), rouges à l'extérieur, jaune crème plus ou moins veiné de rouge à l'intérieur, terminés par un mucron rouge. Involucre jaune, $3 \times 2,5 \mathrm{~mm}$, doliforme. Glandes, 5 , rouge foncé, $1 \times 0,5 \mathrm{~mm}$, en forme de haricot, séparées, lisses, légèrement dressées. Bractées interglandulaires blanc-rose, recouvrant l'ovaire, puis nettement dressées et dépassant les glandes de $1 \mathrm{~mm}$. Fleurs mâles, 5-10; pédicelle blanc $1,5 \mathrm{~mm}$; étamine blanche de $1 \mathrm{~mm} ; 2$ anthères jaunes allongées $0,6 \times 0,4 \mathrm{~mm}$. Fleur femelle apparaissant avant les mâles. Ovaire jaune trilobé $1 \mathrm{~mm}$ diam. Style blanc-vert, $2 \mathrm{~mm}$, droit puis se séparant aux deux tiers en 3 stigmates recourbés, bifides et terminés par deux boules stigmatiques vertes. Fruit blanc-jaune à 3 lobes, les styles sèchent et disparaissent du fruit. Graines non observées. 

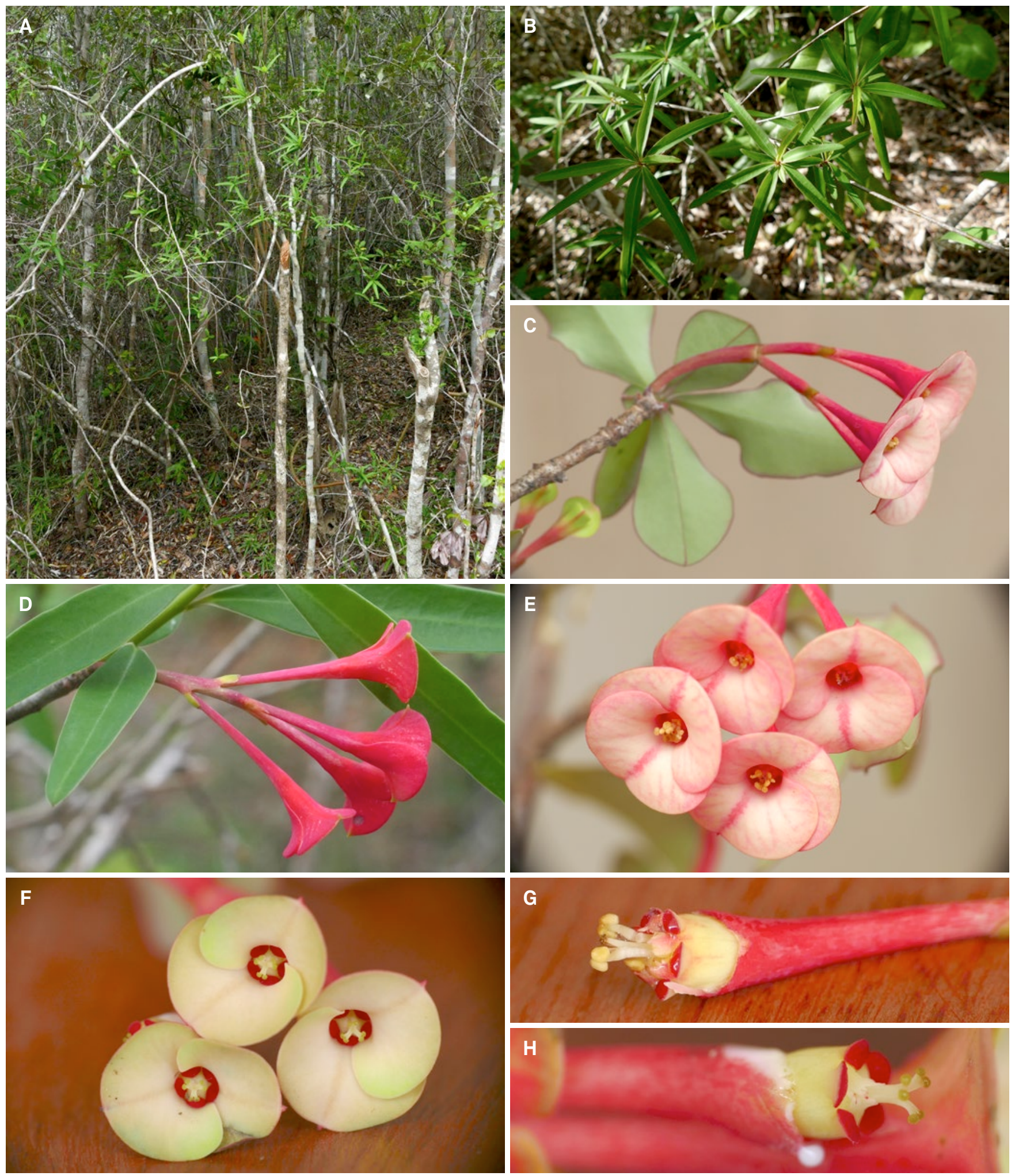

Fig. 1. - Euphorbia longitubicinicyathium J.-P. Castillon \& J.-B. Castillon. A. Port général; B. Rosettes de feuilles;

C. Les feuilles juvéniles et/ou des courts brachyblastes; D. Incyathescence; E. Cyathes à l'anthèse avec les fleurs mâles;

F. Cyathes juvéniles avec la fleur femelle; G-H. Involucre, cyathophylles enlevés.

[Photos: J.-P. Castillon] 
Etymologie. - Cette espèce doit son nom à ses longues cyathes en forme de cor.

Distribution et statut de conservation. - Euphorbia longitubicinicyatbium est connue des forêts denses sèches de Sahafary et Ambolobozobe. Les forêts du bassin des rivières Saharenana et Irodo sont souvent dégradées, soumises à une forte pression humaine et à une forte érosion visible sous forme de lavaka. Ces deux zones sont reconnues importantes pour la conservation des plantes mais non protégées (MBG, 2013; $\mathrm{CEPF}, 2014)$. Avec une zone d'occurrence inférieure à $100 \mathrm{~km}^{2}$, une zone d'occupation de $8 \mathrm{~km}^{2}$ (calculé avec GeoCat; Bachman \& Moat, 2012) et quatre localités connues dans des forêts non protégées et menacées, E. longitubicinicyathium est provisoirement considéré comme «En danger» [EN B1ab (ii,iii,iv,v)+B2ab(ii,iii,iv,v)] selon les Catégories et les Critères de l'UICN (2012).

Notes. - Euphorbia longitubicinicyathium pousse dans des forêts denses et sèches, semi-caducifoliées, formées d'arbustes atteignant 6-8 $\mathrm{m}$ de hauteur, et cet habitat lui a sans doute donné son port si particulier: elle est excessivement grêle, avec des tiges très minces, qui trouvent support sur les arbustes environnants, et se caractérise par son port presque lianescent. En l'absence de support, la tige principale est courbée sous le poids de la plante, et retombe sur le sol; elle est même entièrement couchée sur une longueur de $1 \mathrm{~m}$ sur certains spécimens. La plante a un port très lâche mais possède de nombreuses ramifications. Les tiges secondaires, souvent en position horizontale, reposent sur la végétation environnante, sont longues et minces (diam. de 8-12 mm, plus de $1 \mathrm{~m}$ de longueur), et ont des ramifications très irrégulièrement espacées, parfois des embranchements d'où partent $4-8$ tiges dans toutes les directions, parfois de longues tiges simples, parfois de courts brachyblastes. Les tiges terminales minces et allongées $(10-20 \mathrm{~cm} \times 2-3 \mathrm{~mm})$, sont plutôt lisses et droites, horizontales, et sont terminées par un court morceau de $2-4 \mathrm{~cm}$ de long, redressé, portant des stipules séchées et de nombreuses cicatrices foliaires, puis une rosette terminale de 6 -10 feuilles. Au cours de la croissance des tiges, des feuilles caulinaires se développent, mais elles tombent rapidement et seule une rosette terminale subsiste à la fin. Il est enfin à noter que ces feuilles caulinaires, ainsi que les feuilles juvéniles et parfois celles à l'extrémité des courts brachyblastes possèdent généralement une morphologie différente des feuilles classiques: elles sont plus petites et plus larges $(20-30 \times 10 \mathrm{~mm})$, ovalesrhomboïdales, leur surface est ondulée et non pas lisse, leur marge est rosée et non blanche, leurs stipules sont bien plus petites $(1 \times 0,1 \mathrm{~mm})$.

L'absence d'épines rapproche E. longitubicinicyathium des espèces inermes de Euphorbia sect. Goniostema des forêts de la côte est, par exemple E. geroldii, E. mangelsdorffii, E. robivelonae
(Rauh 1994,1998) et E. thuarsiana (Baillon, 1861). Ces dernières espèces possèdent aussi des incyathescences solitaires formées de quelques cyathes allongés et pendants. La nouvelle espèce reste cependant facile à identifier grâce à des caractères uniques: sa grande taille ( $5 \mathrm{~m}$ vs $1-2 \mathrm{~m}$ pour toutes les autres), la minceur de ses tiges, la forme de ses rosettes de feuilles (rosette terminale en étoile de feuilles allongées lancéolées vs feuilles caulinaires et rosettes lâches de feuilles pyriformes pour E. geroldii, E.thuarsiana et E. mangelsdorffii, feuilles allongées, caulinaires, très nombreuses pour $E$. robivelonae) et la forme et la couleur de ses cyathes $(20 \mathrm{~mm}$, allongés, rouge à l'extérieur, jaune à l'intérieur vs blanc pour $E$. thuarsiana et $E$. robivelonae, rose pâle pour $E$. mangelsdorffi, entièrement rouge pour $E$. geroldii, plus courts et plus ouverts pour ces quatre espèces).

Paratypi. - Madagascar. Prov. Antsiranana: Sahafary, $12^{\circ} 36^{\prime} \mathrm{S} 49^{\circ} 27^{\prime} \mathrm{E}$, 270 m, 26.V.2005, Hong-Wa 207 (CNARP, MO); Sahafary, 12 $36^{\prime}$ S 49 $27^{\prime} \mathrm{E}$, 258 m, 4.XII.2006, Ranaivojaona 1652 (CNARP, MO, P, TAN); Ambolobozobe, Ankonahona, 12³1'S 49³2'E, 27.XI.2007, Rakotonandrasana 1237 (CNARP, MO, P, TAN).

\section{Remerciements}

Tous nos remerciements à James Solomon et Heidi Schmidt (MO) pour la mise à disposition rapide d'images ayant servi à identifier les paratypes. Tous nos remerciements également aux personnes en charge des herbiers de $\mathrm{P}$, de $\mathrm{G}$ et de $\mathrm{K}$ pour les facilités d'accès à leurs bases de données d'échantillons. Merci à Solo Rapanarivo pour son aide dans la consultation de TAN, Roy Gereau (MO) pour sa relecture de la diagnose latine et Martin Callmander pour son aide pour la rédaction et pour le calcul du statut de conservation. 


\section{Références}

Aubriot, X., P. P. Lowry \& T. Haevermans (2014). Taxonomic revision of the Malagasy endemic and enigmatic Euphorbia section Pachysanthae (Euphorbiaceae). Phytotaxa 159: 221-235.

Bachman, S. \& J. Moat (2012). GeoCAT - an open source tool for rapid Red List assesments. Bot. Gard. Conservation Int. J. 9 [http://geocat.kew.org].

Baillon, H. (1861). Species Euphorbiaceorum. Euphorbiacées Africaines. Deuxième partie. Afrique orientale. Adansonia 1: 139-173.

CEPF (2014). Ecosystem profile Madagascar and Indian Ocean islands. Report. Critical Ecosystem Partnership Fund, Conservation International, Washington \& Antananarivo.

Dorsey, B., T. Haevermans, X. Aubriot, J. Morawetz, R. Riina,V. Steinmann \& P. Berry (2013). Phylogenetics, morphological evolution, and classification of Euphorbia subgenus Euphorbia. Taxon 62: 291-315.

MBG (2013). Contribution to status of plant conservation and identification of important gaps. Report to help Ecosystem profileMadagascar. Missouri Botanical Garden, Antananarivo.

RAuH, W. (1994). Euphorbia geroldii, Euphorbia robivelonae, deux espèces nouvelles remarquables du nord-est de Madagascar. Succulentes (France) 1994(1): 5-12.

Rauh, W. (1998). Euphorbia mangelsdorffii, eine neue ZwergEuphorbie aus Madagascar. Kakteen And. Sukk. 49: 44-45.

UICN (2012). Catégories et Critères de la Liste rouge de l'UICN. Version 3.1. Ed.2. Commission de la sauvegarde des espèces de l'UICN, Gland \& Cambridge. 